\title{
Stent Cronus: Chegou o Momento de Adotarmos um Stent Nacional?
}

\author{
Ver artigo relacionado \\ na página 314
}

\author{
Daniel Chamié1, Alexandre Abizaid ${ }^{1}$
}

0 implante de stents para o tratamento percutâneo da doença aterosclerótica coronária obstrutiva, ao prevenir e resolver oclusões agudas do vasoalvo, aumentou a segurança imediata da intervenção coronária percutânea, enquanto no médio prazo mostrou-se irrefutavelmente superior em reduzir as taxas de reestenose $\mathrm{e}^{1,2}$.

A maior aceitação do uso dos stents como modalidade primária para o tratamento percutâneo da doença aterosclerótica coronária ampliou suas aplicações para lesões de maior complexidade. Associado ao crescimento tecnológico, promoveu resposta imediata da indústria, levando ao desenvolvimento de uma grande variedade de modelos de stents vasculares, com desenhos e características inovadoras, tornando-se a modalidade dominante de intervenção coronária percutânea em todo o mundo ${ }^{3}$.

Em teoria, o stent ideal deveria: (1) ser confeccionado com material trombo-resistente e biocompatível; (2) ter adequada flexibilidade em sua forma não-expandida, permitindo a navegação em vasos tortuosos e calcificados; (3) ter adequada radiopacidade, que permita visualização fluoroscópica para guiar seu posicionamento acurado e, no estado expandido, possibilite sua identificação no vaso coronário, facilitando a realização de pós-dilatações e a avaliação de sua perviedade em estudos futuros; (4) apresentar baixo recolhimento elástico e mínimo encurtamento longitudinal quando expandido, mantendo máxima força radial; (5) apresentar configuração que forneça cobertura uniforme da circunferência vascular, gerando baixa relação metal/artéria, boa conformidade com as curvas vasculares, assim como perviedade e acesso a ramos secundários; (6) ter um sistema de entrega flexível e de baixo perfil, de forma a não comprometer a navegabilidade do sistema; (7) ser firmemente montado sobre o balão de entrega, de forma a não ser deslocado do sistema em seu trajeto até o local de implante; (8) ser montado sobre balão de entrega que apresente mínimo crescimento longitudinal $(<1 \mathrm{~mm})$ para fora dos limites do stent, que suporte altas pressões de insuflação sem se romper e que seja nãocomplacente o suficiente para promover expansão previsível e adequada do stent; (9) ter disponibilidade de diâmetros e extensões variados; e (10) apresentar boa relação custo-benefício.

No Brasil, até meados de 2009, estavam registrados na Agência Nacional de Vigilância Sanitária (Anvisa) cerca de 108 stents para uso clínico ${ }^{4}$, nenhum preenchendo plenamente os critérios de "stent ideal", mas muitos ainda sob constante evolução. Diante dessa diversidade de modelos, os stents podem ser classificados de acordo com algumas características estruturais: (1) quanto a sua composição metálica (aço inoxidável, ligas à base de cobalto, tântalo, nitinol); (2) quanto ao revestimento de sua superfície (nenhum, passivo, ativo e biodegradável); (3) quanto à configuração (mola ou coil, anel, estrutura em malha, tubular fenestrado, multidesenho); (4) quanto à forma de acabamento (cortado a laser ou jato d'água); e (5) quanto à forma de liberação (balão-expansível ou autoexpansível).

Nesta edição da Revista Brasileira de Cardiologia Invasiva, Campos et al. ${ }^{5}$ apresentam os resultados clínicos iniciais do primeiro stent de cromo-cobalto fabricado no Brasil. Trata-se de stent balão-expansível, confeccionado com liga metálica de cromo-cobalto L-605, cortado a laser e submetido a tratamento térmico, decapagem química e passivação eletroquímica. Tem desenho em anéis corrugados, com ângulo máximo entre suas hastes de 45 graus, visando à otimização de sua força radial. As hastes são de fina espessura (75 $\mu \mathrm{m} / 0,0030 ")$ e os anéis são construídos em células curtas $(1,1 \mathrm{~mm})$, buscando aumentar sua cobertura circunferencial.

\footnotetext{
1 Instituto Dante Pazzanese de Cardiologia - São Paulo, SP, Brasil.

Correspondência: Alexandre Abizaid. Av. Dr. Dante Pazzanese, 500 - Ibirapuera - São Paulo, SP, Brasil - CEP 04012-180

E-mail: aabizaid@uol.com.br

Recebido em: 18/9/2009 • Aceito em: 21/9/2009
} 
Podem essas características influenciar a eficácia desse novo stent? Certamente sim. A maioria dos stents coronários testados clinicamente é confeccionada a partir de ligas metálicas, sendo o aço inoxidável a mais frequentemente utilizada. O aço é predominantemente composto de ferro, que é biologicamente inerte, mas também contém cerca de $12 \%$ de níquel e $2 \%$ de molibdênio, substâncias que, em indivíduos alérgicos, podem contribuir para aumento do risco de reestenose intrastent ${ }^{6}$. Ligas de cromo-cobalto permitiram a confecção de stents com hastes mais finas sem ocasionar prejuízo a sua radiopacidade e força radial ${ }^{7}$.

Características da superfície do stent também podem ter influência no desempenho dessas próteses. Estudos in vitro sugerem que metais que possuem maior potencial de superfície atraem plaquetas carregadas negativamente e proteínas plasmáticas, conferindo trombogenicidade aumentada ${ }^{8}$. Uma superfície rugosa também pode promover trombose dos stents ${ }^{9}$. Nesse sentido, polimento eletroquímico dos stents metálicos tem resultado em superfícies menos trombogênicas, meIhorando sua segurança ${ }^{10}$. Várias coberturas também já foram aplicadas à superfície dos stents, visando à redução da trombogenicidade ou da reestenose dos stents metálicos. No entanto, na prática, a maioria dessas coberturas não conferiu vantagens comparativamente aos stents metálicos descobertos ${ }^{11-13}$.

Quanto a sua configuração geométrica, os stents em formato de mola (coil), utilizados no passado, quando comparados aos stents de tubos fenestrados, demonstraram baixa força radial, maior recolhimento elástico e prolapso de placa, mais dissecções de bordas, menor diâmetro luminal mínimo inicial e maiores incidências de oclusão aguda e reestenose tardia ${ }^{14}$. Os stents em tubos fenestrados, cujo protótipo foi o Palmaz-Schatz, aumentaram a força radial e a cobertura circunferencial do vaso. No entanto, os modelos iniciais tinham alto perfil e baixa flexibilidade, melhorados com a manufatura de stents cortados a laser a partir de tubos metálicos em desenhos específicos e com a introdução dos stents multicelulares. Os stents híbridos ou modulares aumentaram ainda mais a flexibilidade e a capacidade de entrega dos stents tubulares pela adição de pontes flexíveis unindo os pequenos pedaços de módulos repetidos.

O desenho dos stents pode ser subclassificado como: (1) células abertas, de tamanhos e formas variadas ao longo do stent, aumentando sua flexibilidade, entrega, conformatividade e acesso a ramos secundários, porém com cobertura circunferencial menos homogênea e maior possibilidade de prolapso de placa; (2) células fechadas, incorporando um padrão unicelular repetido e fornecendo cobertura circunferencial mais uniforme e menos prolapso de placa (redução da flexibilidade e acesso a ramos laterais são suas limitações); e (3) intermediário, incorporando as duas características anteriores, de forma a manter boa cobertura circunferencial, alta flexibilidade e acesso aos ramos secundários. Já foi previamente demonstrado que o desenho e a geometria dos stents têm impacto significativo em seus resultados de longo prazo ${ }^{15}$.

Por fim, a redução da espessura das hastes dos stents talvez tenha sido o avanço tecnológico que mais impacto apresentou na redução das taxas de reestenose ${ }^{16,17}$. Estudo recente com tomografia por convergência óptica intracoronária demonstrou que a espessura das hastes e a geometria das células do stent podem influenciar a adequada aposição do stent à parede vascular agudamente, embora sem correlação com eventos adversos ao final de 12 meses $^{18}$.

Assim, no que se refere a sua composição metálica e aos aspectos físicos e estruturais, o stent Cronus agrega características que, comprovadamente, podem influenciar de forma positiva sua efetividade. Posicionase, portanto, em condições de competir com os stents mais utilizados na prática contemporânea. A Tabela 1 apresenta as principais características desse novo stent coronário em relação a alguns stents disponíveis comercialmente.

Qual o impacto dessas características na eficácia e nos desfechos clínicos do novo stent Cronus? O stent Cronus foi testado em 53 pacientes portadores de doença aterosclerótica coronária, incluindo desde quadros estáveis até infarto agudo do miocárdio. A média das idades foi de 62 anos, com $26 \%$ de diabéticos. O diâmetro médio de referência do vaso-alvo foi de 2,7 \pm 0,7 mm e a extensão das lesões, de 16,7 \pm $3,5 \mathrm{~mm}$. Foi permitido o tratamento de enxertos venosos $(2 \%)$. Os autores encontraram perda tardia média de $0,7 \pm 0,5 \mathrm{~mm}$ e taxa de reestenose angiográfica de $17,5 \%$, seis a nove meses após o procedimento índice. Aos 12 meses, a taxa de eventos maiores foi de $24,4 \%$, constituídos por $7,5 \%$ de óbito $(n=4$, dois de origem cardíaca), 5,6\% de infarto agudo do miocárdio não-fatal (todos periprocedimento) e 11,3\% de nova revascularização do vaso-alvo. Esses resultados são consistentes e comparáveis àqueles de registros que avaliaram a segurança e a eficácia de stents contemporâneos disponíveis para uso clínico (Tabela 1).

No entanto, esse registro merece algumas considerações. Esse é um estudo clínico de fase I, que segue o caminho das boas práticas da pesquisa clínica, precedido por adequada avaliação pré-clínica em animais. Entretanto, para uma análise do tipo First-inMan, que visa a avaliar a segurança e a eficácia de um novo stent em humanos, a liberalidade da inclusão de pacientes portadores de infarto agudo do miocárdio e tratamento de enxertos de veia safena, mesmo que em pequeno número, confere um desenho arrojado a esse estudo. Potencialmente, desfechos adversos podem ocorrer em função da complexidade clínica desses pacientes, e não propriamente por falência do dispositivo. A realização de reestudo angiográfico em ape- 


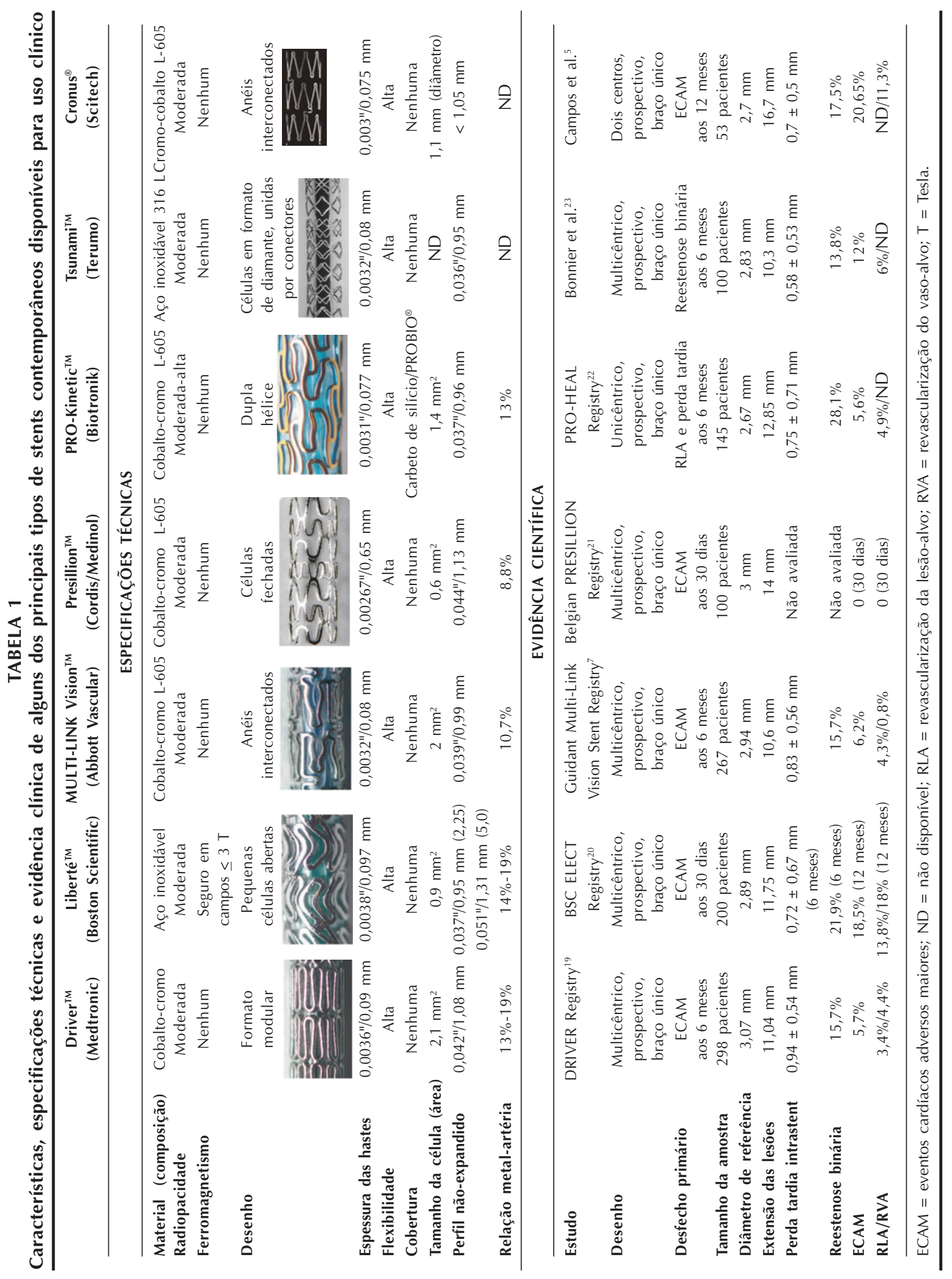


nas $58,5 \%$ da população tratada, de forma não préespecificada, é a principal limitação desse estudo. Em estudos de fase I, cujo principal objetivo é avaliar a segurança do medicamento/dispositivo em investigação, bem como se o mesmo apresenta efeitos colaterais que impossibilitem seu uso clínico, análise angiográfica detalhada de todos os pacientes incluídos agregaria mais informação e seria fundamental. Além disso, para análise de sua eficácia, o tamanho da amostra é insuficiente para a verificação dos efeitos desse novo stent sobre as taxas de reestenose e necessidade de novas revascularizações. Embora a utilização de desfechos angiográficos substitutos para reestenose seja controversa, índices angiográficos como perda luminal tardia e porcentual de estenose intrastent no seguimento a médio prazo mostraram-se marcadores substitutos adequados para determinação da eficácia clínica do stent investigado, com a vantagem de necessitar tamanho de amostra significativamente reduzido ${ }^{24}$. Permitem, ainda, uma comparação mais objetiva com os demais stents disponíveis. Nesse sentido, dispor dos dados angiográficos de todos os pacientes incluídos seria relevante. $\mathrm{O}$ indicador clínico mais comumente utilizado para aferir a eficácia de um stent é a revascularização da lesão-alvo ${ }^{24}$. Apresentação somente das taxas de revascularização do vaso-alvo não permite diferenciar se a necessidade de novas revascularizações ocorreu por falência do novo dispositivo ou por progressão da doença em outros segmentos do vaso-alvo. Torna-se, portanto, altamente recomendável a realização de estudo randomizado, a princípio com desfecho angiográfico documentado em número mais expressivo de pacientes. Por fim, vale ressaltar a não-utilização do ultrassom intracoronário nesse estudo.

No Brasil, o custo dos stents farmacológicos e o não-reembolso de seu valor pelo Sistema Único de Saúde fazem com que os stents não-farmacológicos ainda sejam os dispositivos mais amplamente utilizados. A demonstração do bom desempenho do stent Cronus e da adequada relação custo-efetividade podem tornar esse dispositivo altamente competitivo. Suas características estruturais, com hastes de fina espessura e geometria única, parecem ser bastante adequadas para sua utilização como plataforma em stents farmacológicos. Apresenta potencial de gerar baixo grau de injúria à parede do vaso e permitir distribuição homogênea do fármaco. A comprovação de boa flexibilidade e navegabilidade desse dispositivo pode permitir, ainda, sua utilização em vasos com anatomia mais complexa.

À medida que crescerem as evidências clínicas e científicas a respeito do stent Cronus, acreditamos que esse dispositivo de fabricação nacional terá o potencial de ser gradualmente incorporado a nossa prática clínica.

\section{CONFLITO DE INTERESSES}

Os autores declararam inexistência de conflito de interesses.

\section{REFERÊNCIAS BIBLIOGRÁFICAS}

1. Serruys PW, de Jaegere P, Kiemeneij F, Macaya C, Rutsch W, Heyndrickx G, et al. A comparison of balloon-expandablestent implantation with balloon angioplasty in patients with coronary artery disease. Benestent Study Group. N Engl J Med. 1994;331(8):489-95.

2. Fischman DL, Leon MB, Baim DS, Schatz RA, Savage MP, Penn I, et al. A randomized comparison of coronary-stent placement and balloon angioplasty in the treatment of coronary artery disease. Stent Restenosis Study Investigators. N Engl J Med. 1994;331(8):496-501.

3. Topol EJ. Coronary-artery stents: gauging, gorging, and gouging. N Engl J Med. 1998;339(23):1702-4.

4. Stents farmacológicos e stents metálicos no tratamento da doença arterial coronariana. Boletim Brasileiro de Avaliação de Tecnologias em Saúde (BRATS), número 8, Junho 2009. Disponível em: www.anvisa.gov.br/divulga/newsletter/brats/ 2009/BRATS8.pdf. Acesso em 6/setembro/2009.

5. Campos CAHM, Ribeiro EE, Lemos PA, Obregon A, Ribeiro $H$, Spadaro AG, et al. Resultados clínicos iniciais do primeiro stent de cromo-cobalto concebido no Brasil. Rev Bras Cardiol Invas. 2009;17(3):314-9.

6. Köster R, Vieluf D, Kiehn M, Sommerauer M, Kähler J, Baldus S, et al. Nickel and molybdenum contact allergies in patients with coronary in-stent restenosis. Lancet. 2000; 356(9245):1895-7.

7. Kereiakes DJ, Cox DA, Hermiller JB, Midei MG, Bachinsky WB, Nukta ED, et al., for the Guidant Multi-Link Vision Stent Registry Investigators. Usefulness of a cobalt chromium coronary stent alloy. Am J Cardiol. 2003;92(4):463-6.

8. Baier RE, Dutton RC. Initial events in interactions of blood with a foreign surface. J Biomed Mater Res. 1969;3(1):191-206.

9. Zitter H, Plenk H Jr. The electrochemical behavior of metallic implant materials as an indicator of their biocompatibility. J Biomed Mater Res. 1987;21(7):881-96.

10. De Scheerder I, Sohier J, Wang K, Verbeken E, Zhou XR, Frooyen L, et al. Metallic surface treatment using electrochemical polishing decreases thrombogenicity and neointimal hyperplasia after coronary stent implantation in a porcine model. Eur Heart J. 1997;18 Suppl:153.

11. Kastrati A, Schömig A, Dirschinger J, Mehilli J, von Welser $\mathrm{N}$, Pache J, et al. Increased risk of restenosis after placement of gold-coated stents: results of a randomized trial comparing gold-coated with uncoated steel stents in patients with coronary artery disease. Circulation. 2000;101(21):2478-83.

12. Wöhrle J, Al-Khayer E, Grötzinger $U$, Schindler C, Kochs $M$, Hombach V, et al. Comparison of the heparin coated vs. the uncoated Jostent: no influence on restenosis or clinical outcome. Eur Heart J. 2001;22(19):1808-16.

13. Haase J, Störger $H$, Hofmann M, Schwarz CE, Reinemer $H$, Schwarz F. Comparison of stainless steel stents coated with turbostratic carbon and uncoated stents for percutaneous coronary interventions. J Invasive Cardiol. 2003;15(10):562-5.

14. Lansky AJ, Roubin GS, O'Shaughnessy CD, Moore PB, Dean LS, Raizner AE, et al. Randomized comparison of GR-II stent and Palmaz-Schatz stent for elective treatment of coronary stenoses. Circulation. 2000;102(12):1364-8.

15. Kastrati A, Dirschinger J, Boekstegers P, Elezi S, Schühlen $H$, Pache J, et al. Influence of stent design on 1-year outcome after coronary stent placement: a randomized comparison of five stent types in 1,147 unselected patients. Catheter Cardiovasc Interv. 2000;50(3):290-7.

16. Kastrati A, Mehilli J, Dirschinger J, Dotzer F, Schühlen H, Neumann FJ, et al. Intracoronary stenting and angiographic results: strut thickness effect on restenosis outcome (ISARSTEREO) trial. Circulation. 2001;103(23):2816-21. 
17. Pache J, Kastrati A, Mehilli J, Schühlen H, Dotzer F, Hausleiter $J$, et al. Intracoronary stenting and angiographic results: strut thickness effect on restenosis outcome (ISAR-STEREO II) trial. J Am Coll Cardiol. 2003;41(8):1283-8.

18. Tanigawa J, Barlis P, Dimopoulos K, Dalby M, Moore P, Di Mario $C$. The influence of strut thickness and cell design on immediate apposition of drug-eluting stents assessed by optical coherence tomography. Int J Cardiol. 2009;134(2):180-8.

19. Sketch MH Jr, Ball M, Rutherford B, Popma JJ, Russel C, Kereiakes DJ, on behalf of the Driver Investigators. Evaluation of the Medtronic (Driver) cobalt-chromium alloy coronary stent system. Am J Cardiol. 2005;95(1):8-12.

20. Boston Scientific Corporation. Liberté ${ }^{\circledR}$. 2009. Disponível em: http://www.bostonscientific.com

21. Legrand $V$. The Belgian PRESILLION Registry: 30-day followup. Cardiac \& Vascular Update. 2009;2:30-2.
22. Dahm JB, Willems T, Wolpers HG, Nordbeck H, Becker J, Ruppert J. Clinical investigation into the observation that silicon carbide coating on cobalt chromium stents leads to early differentiating functional endothelial layer, increase safety and DES-like recurrent stenosis rates: results of the PRO-Heal Registry (PRO-Kinetic enhancing rapid in-stent endothelialisation). Eurolntervention. 2009;4(4):502-8.

23. Bonnier HJRM, van den Heuvel P, Legrand V, Tanabe K, Vos J, Serruys PW. Clinical and angiographic outcomes after Tsunami $^{\mathrm{TM}}$ coronary stent placement. J Invas Cardiol. 2004; 16(5):252-6.

24. Pocock SJ, Lansky AJ, Mehran R, Popma JJ, Fahy MP, Na Y, et al. Angiographic surrogate end points in drug-eluting stent trials: a systematic evaluation based on individual patient data from 11 randomized, controlled trials. J Am Coll Cardiol. 2008;51(1):23-32. 